\title{
La formación docente y el uso de técnicas activas en la educación superior
}

\section{Teacher training and the use of active techniques in higher education}

\author{
Gino Iván Ayón Ponce ${ }^{1}$ \\ ginoayon1@hotmail.com \\ Amparo Bienvenida Baque Morán ${ }^{2}$ \\ amparo.baque@unesum.edu.ec \\ Miguel Ángel Jaime Baque ${ }^{3}$ \\ angel.m.86@hotmail.com \\ Paco Egdon Granoble Chancay ${ }^{4}$ \\ paco.granoble@unesum.edu.ec \\ Carlos Artemidoro Zea Barahona ${ }^{5}$ \\ caryfran3@hotmail.com \\ Erick Geovanny Salazar Ponce ${ }^{6}$ \\ erick.salazar@unesum.edu.ec
}

Recibido: 1/09/2017, Aceptado: 1/11/2017

\begin{abstract}
RESUMEN nivel de cognitivo de los estudiantes.

\footnotetext{
1 Docente de la Universidad Estatal del Sur de Manabí. Ecuador

2 Docente de la Universidad Estatal del Sur de Manabí. Ecuador

3 Docente de la Universidad Estatal del Sur de Manabí. Ecuador

4 Docente de la Universidad Estatal del Sur de Manabí. Ecuador

5 Docente de la Universidad Estatal del Sur de Manabí. Ecuador

6 Docente de la Universidad Estatal del Sur de Manabí. Ecuador
}

Este artículo es el resultado de un trabajo de investigación, reflejado en la importancia de conocer el manejo didáctico y pedagógico aplicado de la enseñanza aprendizaje en la Educción Superior. El objetivo fue identificar cual es la formación docente y su aplicación de técnicas activas que inciden en el interaprendizaje de los estudiantes de la Carrera de Comercio Exterior de la Universidad Estatal del Sur de Manabí. Se abordó una metodología basada en una investigación de campo acompañada de métodos y técnicas para la recopilación de la información, mediante la encuesta se evidenció el déficit educativo en la enseñanza de educación superior, la misma que arrojo resultados relevantes de la problemática sobre el limitado uso de técnicas en el desarrollo de la clase y se concluye con un tutorial didáctico de técnicas activas para potenciar el proceso enseñanzas aprendizaje. Esta investigación permite fortalecer el

Revista científica Ciencia y Tecnología Vol 18 No 17 págs. 273-291 http://cienciaytecnologia.uteg.edu.ec 


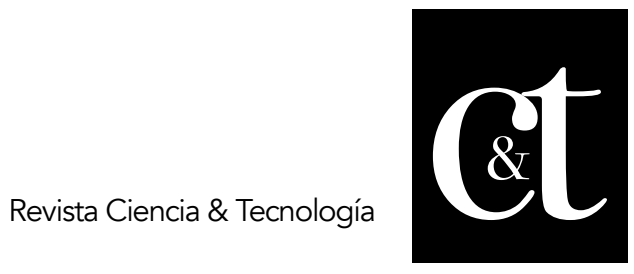

No. 17, 31 de enero de 2018

ISSN impreso: 1390 - 6321

Palabras clave: Formación docente, técnicas, educación superior, enseñanza, aprendizaje

\begin{abstract}
This article is the result of a research work, reflected in the importance of knowing the didactic and pedagogical application of teaching teaching in Higher Education. The objective was to identify what is the teacher training and its application of active techniques that affect the interlearning of the students of the Foreign Trade Program of the State University of the South of Manabí. A methodology based on a field investigation accompanied by methods and techniques for the collection of information through the survey where the educational deficit in the higher education teaching was evidenced, the same one that showed relevant results of the problem about the limited use of techniques in the development of the class and concludes with a didactic tutorial of active techniques to enhance the learning teaching process. This research allows to strengthen the cognitive level of students.
\end{abstract}

Keywords: Teacher training, techniques, higher education, teaching, learning

\title{
Introducción
}

La formación docente es un aspecto muy importante, en el que se toma en consideración un sinnúmero de aspectos que se requieren para llegar a alcanzar las metas preestablecidas en el campo de la educación superior, aplicando técnicas activas de calidad, ya sean cognitivas, procedimentales, conceptuales y actitudinales que permitan afianzar las metas y llegar a los educandos con una educación calibrada basada en los ejes transversales para lograr un aprendizaje significativo, acorde al tiempo, espacio y ubicación geográfica de los estudiantes, tomando en cuenta las necesidades, pero más aún sus requerimientos de capacidades intelectuales. Todo esto con la perspectiva de responder con pertinencia ante la sociedad con una educación activa y participativa.

De acuerdo a Hornilla (1999), la formación docente del profesorado ha sido una cuestión poco tratada en la universidad, pues siempre existió la creencia de que para enseñar bien es suficiente con tener un buen dominio de la disciplina que se imparte al alumnado. Hoy, ese viejo prejuicio ha entrado en crisis. En este trabajo se ofrecen distintas alternativas, tanto teóricas como prácticas, que abordan la formación del profesorado universitario desde perspectivas pedagógicas plurales.

Madrid (2005, p. 23), en su investigación realizada en el Espacio Europeo de Educación Superior, indica que el profesorado universitario tenga una formación docente adecuada. Los responsables administrativos y académicos de la universidad tienen que planificar y financiar esa formación. También, es necesario que la universidad valore e incentive la buena docencia (de la misma manera que ya lo viene haciendo con los méritos investigadores del profesorado). Para ello es necesaria una evaluación de la formación y una evaluación docente del profesorado. 


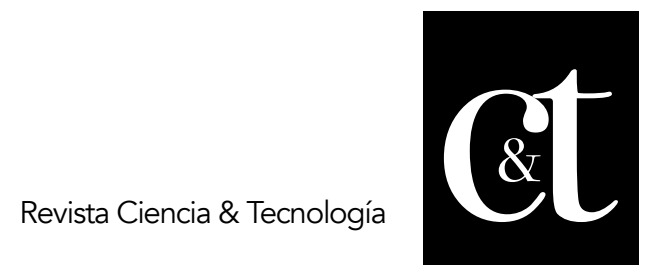

No. 17, 31 de enero de 2018

ISSN impreso: 1390 - 6321

Según Comenius (2009), "las técnicas activas son, en general, procedimientos que buscan obtener través de una secuencia determinada de pasos o comportamientos, uno o varios productos precisos. Determinan de manera ordenada la forma de llevar a cabo un proceso, sus pasos definen claramente cómo ha de ser guiado el curso de las acciones para conseguir los objetivos propuestos" (p. 4).

Con lo expuesto por los autores, la formación docente y las técnicas dentro del interaprendizaje permiten a los estudiantes realizar actividades que propicien la indagación de sus ideas intelectuales y que desarrollen su pensamiento crítico. El alumno debe ser gestor de su propio aprendizaje, a través de la experiencia de reflexión educativa donde los participantes aportan su experiencia particular enriqueciendo y ampliando la experiencia colectiva.

Como podemos mencionar, la excelente formación docente y la aplicación de las técnicas activas para el perfeccionamiento de las capacidades de los educandos, con un aprendizaje más atrayente y motivantes. De esta manera se logra alcanzar una educación participativa en los estudiantes de la carrera de Comercio Exterior.

\section{La Formación y la práctica docente}

Si bien la reflexión sobre la relación de la teoría con la práctica en la formación docente excede el propósito del presente trabajo, no puede dejar de señalarse que las distintas tradiciones suponen una forma particular de aquella relación. Así, en la tradición eficientista, puede observarse la concepción de creer posible que la práctica pedagógica pueda producirse en un contexto teórico y luego ser aplicada en situaciones prácticas. Es decir, derivar de la teoría científica reglas que aseguren resultados y conformar la práctica docente en la mera aplicación instrumental: "(...) así entendida, la teoría de la educación es una forma de ciencia aplicada que emplea generalizaciones empíricamente comprobadas como una base para resolver problemas educativos y guiar la práctica (...)" (Carr, 1990, p. 75).

Se ha observado que la corriente de carácter eficientista ha intentado escindir los ámbitos de planificación y ejecución, con el que el docente pierde virtualmente la reflexión sobre su propia tarea. Cuanto más se separan estos ámbitos tanto más intensos es el proceso de descualificación del trabajo docente, y más dependiente se los hace de los "especialistas" (Apple \& Jungck, 1990). En tal sentido, aceptar que toda práctica no se encuentra completa si no incluye lo que el "Práctico" piensa de ella es tan necesario como aceptar que ningún teórico puede hacer una observación teóricamente neutral (Ídem).

La relación con el conocimiento no responde a la repetición textual de una receta pedagógica sino que es una resultante de múltiples factores: la propia experiencia que el docente tuvo como alumno la reinterpretación que hace de teorías o fragmentos de teorías, como así también de las posibilidades de implementación que su contexto, y sus circunstancias, le permiten en este sentido, diversas investigaciones concluyen que "(...) el docente no es un sujeto neutro que aplica técnicas sino un sujeto cargado de supuestos, creencias, valores e ideas que determinan no sólo las formas de ejercer 


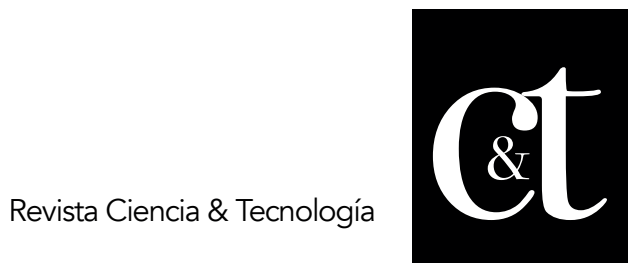

No. 17, 31 de enero de 2018

ISSN impreso: 1390 - 6321

su rol sino también los resultados de la enseñanza. Estas concepciones operan como "teorías prácticas" basadas en juicios empíricos, fruto de sus experiencias escolares como alumno y, después como docente" (Davini \& Alliaud, 1995, p. 117).

En tal sentido, en la formación docente necesariamente deberá incluirse: los enfoques relevantes en cada campo disciplinar, incluyendo los más actuales y su potencial evolución; la explicitación de la estructuración temática del campo; una selección de temas del campo que combine su perfil de especialización con lo dicho respecto del saber de fundamento, y que tenga en cuenta que se trata de un trabajo académico de adquisición de un saber disciplinar donde se incluye el desarrollo de competencias para su tratamiento pedagógico (Sánchez de Magurno, 1998, p. 143-144).

\section{Técnicas Activas}

Según Rosler (2007), "Las técnicas de enseñanza son instrumentos en un proceso de formación educativo, permitiendo explorar sus ideas y pensamientos. Las técnicas activas deben ser participativas para realmente generar un proceso de aprendizaje como el que se plantea, porque permiten: Desarrollar un proceso colectivo de discusión y reflexión, colectivizar un conocimiento individual, enriquecer éste y potenciar realmente el conocimiento colectivo. Permiten una creación colectiva del conocimiento donde todos somos participes de su elaboración y, por lo tanto, también de sus implicancias prácticas determinado, ya sea en el campo de la ciencia, de la tecnología, del arte, del deporte, de la educación o en cualquier otra actividad" ( $p$. 289).

Las técnicas activas que forman parte de la didáctica se conciben como el conjunto de actividades que el maestro estructura para que el estudiante construya el conocimiento, lo transforme, lo problematice, y lo evalúe, además de participar junto con el estudiante en la recuperación de su propio proceso (Pazmiño, 2011, p. 12).

Las técnicas activas involucran al estudiante como actor principal del proceso de aprendizaje, quien desarrolla habilidades de búsqueda, selección, análisis y evaluación (López, 2003, p. 12, 14).

Haciendo énfasis a las definiciones de técnicas activas se evidencia que son indispensables en el proceso de aprendizaje instrumentos que el docente utiliza para planificar, facilitar la construcción del conocimiento cognitivo constructivista, promoviendo la investigación, el análisis de la información y la obtención de sus propias conclusiones.

Tobon (2005) manifiesta que la técnica es considerada como un procedimiento didáctico que se presta a ayudar a realizar una parte del aprendizaje que se persigue con la estrategia.

Así mismo el autor señala que la técnica didáctica es también un procedimiento lógico y con fundamento psicológico destinado a orientar el aprendizaje del alumno. Lo puntual de la técnica es que ésta incide en un sector específico o en una fase del curso 
o tema que se imparte, como la presentación al inicio del curso, el análisis de contenidos, la síntesis o la crítica del mismo. La técnica didáctica es el recurso particular de que se vale el docente para llevar a efecto los propósitos planeados desde la estrategia.

Las técnicas didácticas que indica Flores (1994), en la Revista de Investigación Educativa en el análisis de los "Fundamentos pedagógicos, estrategias de enseñanza y técnicas didácticas", son:

"Las técnicas didácticas representan un conjunto de actividades ordenadas y articuladas dentro del proceso de enseñanza-aprendizaje de una materia. Con base en ellas se puede organizar totalmente un curso o ciertos temas o contenidos específicos del mismo" (Ibídem, p. 2).

Las técnicas didácticas son aquellas que permiten concretar el conocimiento en los estudiantes mediante la didáctica y son orientadas a mejorar la forma de la enseñanza y el aprendizaje con la aplicación de estrategias como la técnica lluvia de ideas, la dramatización, la exposición, y toda actividad que sea didáctica. Estas son parte relevante del quehacer educativo, potenciando el proceso de la enseñanza en la vida diaria del educando, guiando con claridad los objetivos durante todo el proceso y lograr que la clase sea dinámica, activa y creativa acorde a las necesidades del estudiante.

\section{Clasificación de Técnicas Activas de Aprendizaje}

La relevancia de realizar un trabajo eficiente en el quehacer educativo está en la aplicación de técnicas activas de aprendizaje, basada en la organización y las actividades de aprendizaje que el Consejo de Educación Superior del Ecuador propone en su Reglamento de Régimen Académico.

Tal como lo señala el Art. 10: La organización del aprendizaje consiste en la planificación del proceso formativo del estudiante, a través de actividades de aprendizaje con docencia, de aplicación práctica y de trabajo autónomo, que garantizan los resultados pedagógicos correspondientes a los distintos niveles de formación y sus modalidades.

Desde esta perspectiva el profesor debe aplicar técnicas activas en su jornada pedagógica.

Estudio de Casos:

Es una Técnica Didáctica Activa que presenta a los aprendices un episodio o situación concreta dando amplia información sobre las circunstancias que lo envuelven con el fin de analizarlo, resolverlo, elaborar conclusiones y proponer posibles vías de actuación para su resolución.

La expansión progresiva de esta técnica surgida en Estados Unidos durante la segunda década del siglo XX en la formación de universitarios en estudios 
empresariales y jurídicos ha dado lugar a una diversificación de enfoques u orientaciones sobre el tipo de casos que se pueden aplicar en la formación. Siguiendo La clasificación del Instituto ITESM (Instituto Tecnológico de Monterrey, 2010) podemos hablar de tres modelos:

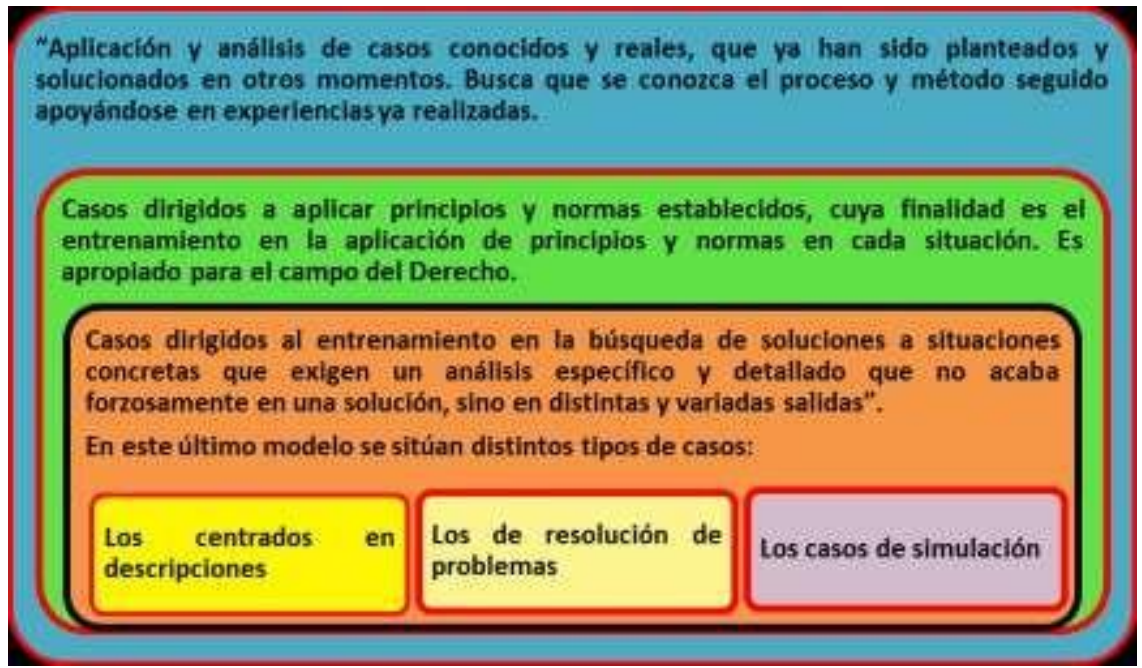

\section{Gráfico 1. Modelo de Estudio de Casos}

Elaborado por: Grupo investigador

Como señala Aznar (1995), su aplicación en el aula, requiere una importante y precisa planificación y preparación por parte del profesor. Este trabajo comienza con la identificación de Casos susceptibles de ser llevados al aula, y que tengan potencial para el desarrollo de los diferentes objetivos/resultados que se han identificado para cada proceso formativo.

Siguiendo a diversos autores (Aguirre, Aznar, ITESM) podemos señalar las siguientes fases como parte de la secuencia didáctica para desarrollar en el aula el Estudio de un Caso:

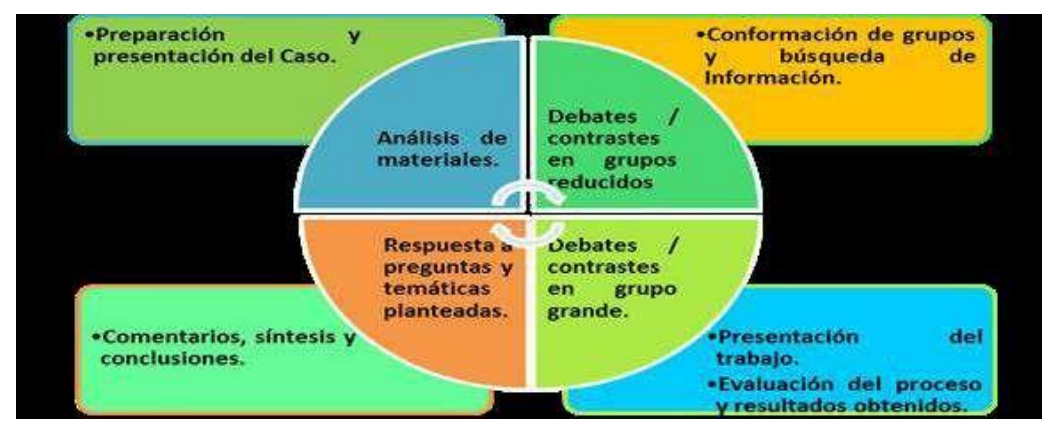

Gráfico 2. Fases de secuencia didáctica del Estudio de Caso Elaborado por: Grupo investigador 
Juegos de Simulación:

Los Juegos de Simulación son otra de las técnicas didácticas activas que se vienen utilizando en procesos formativos muy diversos, donde se define los mismos como "reproducciones simplificadas de la vida real en las que se elimina la mayor parte de la información irrelevante, se secuencian las fases y se permite a los aprendices ser los verdaderos actores de la situación, enfrentándose a la necesidad de tomar decisiones y valorar sus resultados" (Martín, 2013).

En los Juegos de Simulación, "la situación se resuelve a partir de la representación de una serie de papeles o roles que adopta el alumnado. La interpretación de estos papeles se hace en base a una serie de reglas de juego y al perfil que se ha definido para cada personaje" (Carrera \& Otros, 2006).

En este sentido, plantean la posibilidad de uso de los Juegos de Simulación en tres grandes momentos, al inicio de una secuencia de aprendizaje, durante el desarrollo de la misma, o al finalizar dicho proceso.

Aunque no existe una secuencia única en la aplicación de la técnica, pues depende del juego mismo, sí que con carácter general pueden señalarse las siguientes fases que están siempre presentes en todos los Juegos de Simulación:

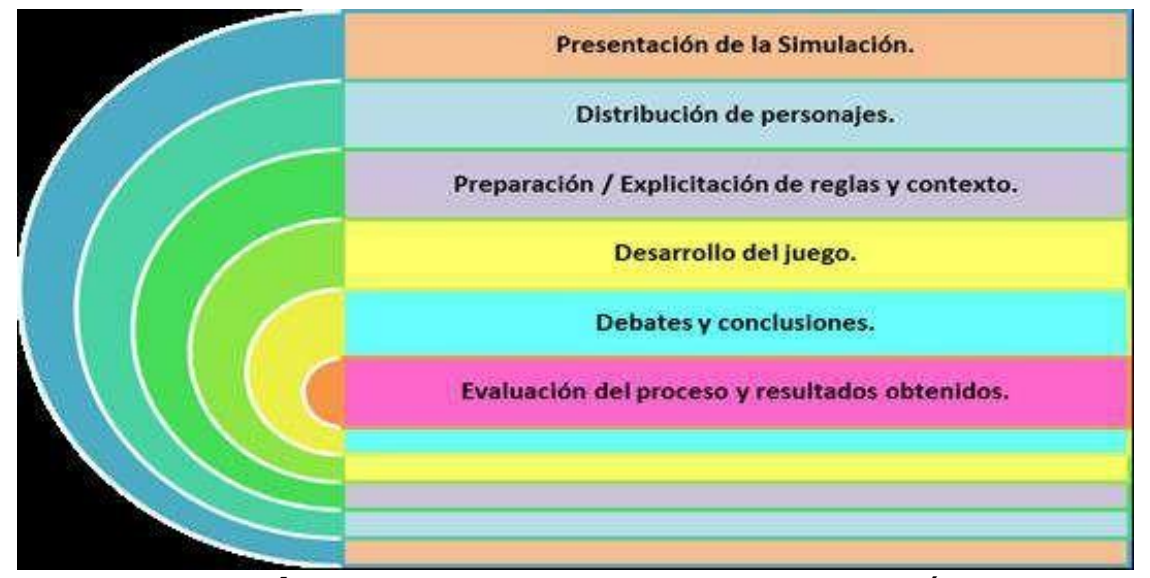

Gráfico 3. Fases de Juegos de Simulación

Elaborado por: Grupo investigador

Aprendizaje basado en problemas:

Prieto (2006, p. 173-196), defendiendo el enfoque de aprendizaje activo señala que "el aprendizaje basado en problemas representa una estrategia eficaz y flexible que, a partir de lo que hacen los estudiantes, puede mejorar la calidad de su aprendizaje universitario en aspectos muy diversos". Así, el ABP ayuda al alumno a desarrollar y a trabajar diversas competencias: 


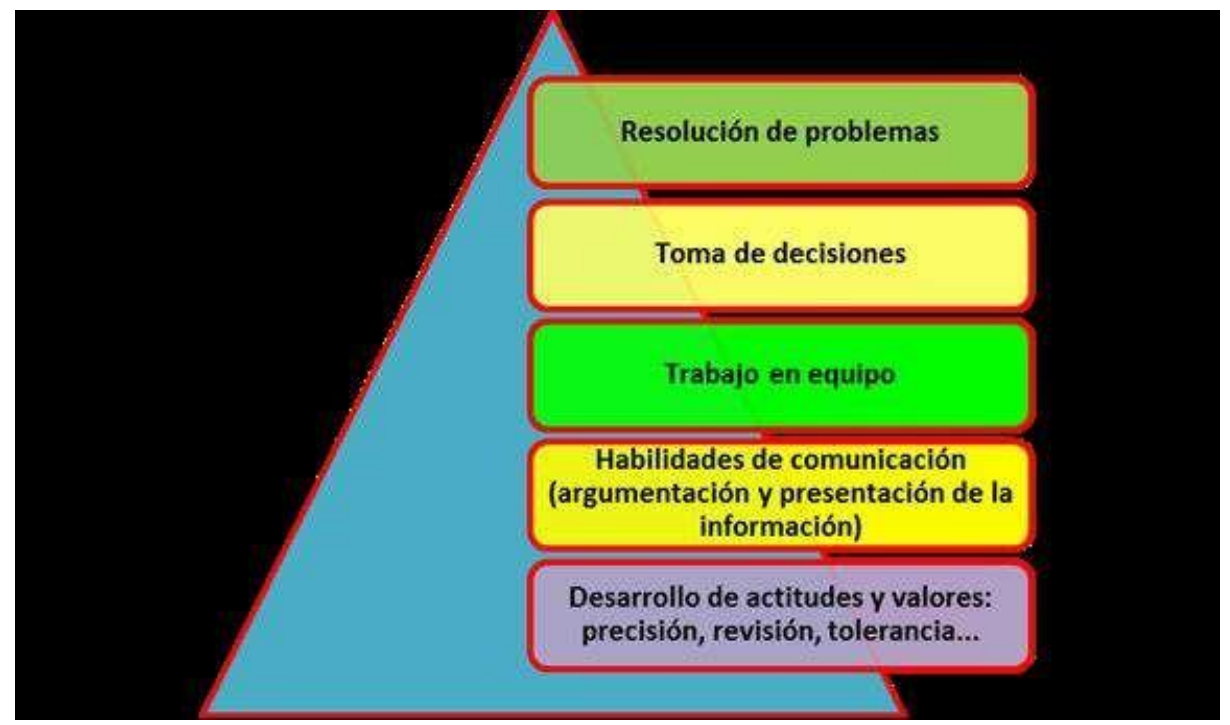

Gráfico 4. Enfoque del aprendizaje basado en problemas Elaborado por: Grupo investigador

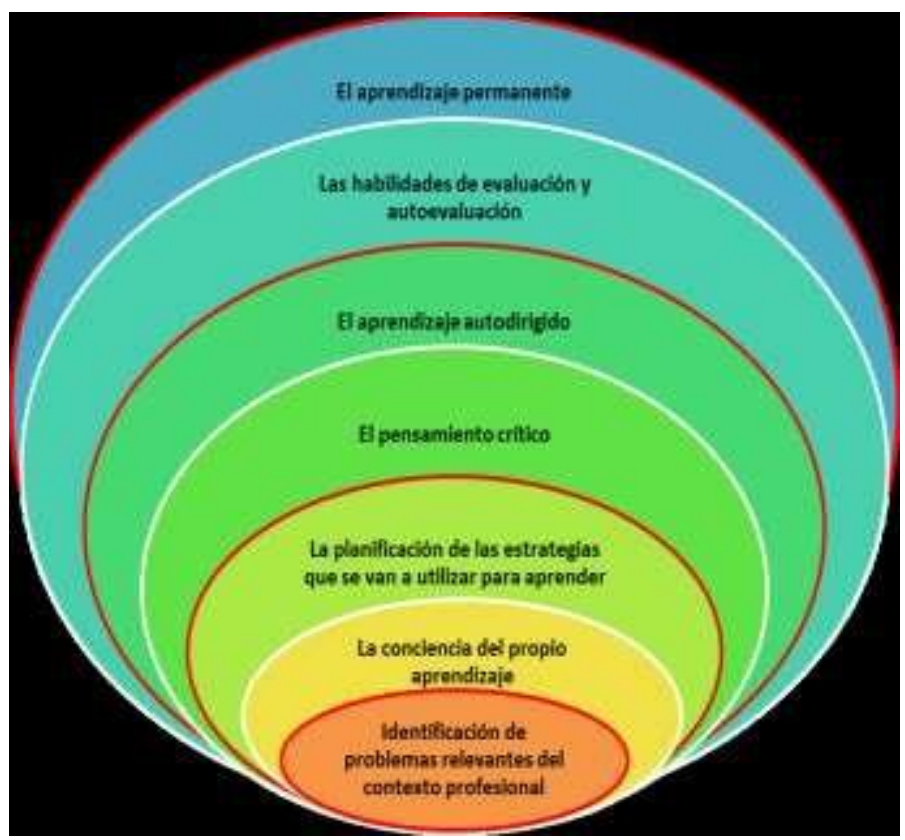

\section{Gráfico 5. Competencia del aprendizaje basado en problemas} Elaborado por: Grupo investigador 
Del mismo modo, Benito \& Cruz (2005), aparte de las competencias ya citadas, indican que el ABP favorece el desarrollo del razonamiento eficaz y la creatividad.

Otras técnicas didácticas:

Además de las técnicas mencionadas hasta el momento, existen otras muchas que, en general, requieren ser utilizadas en combinación con las anteriores:

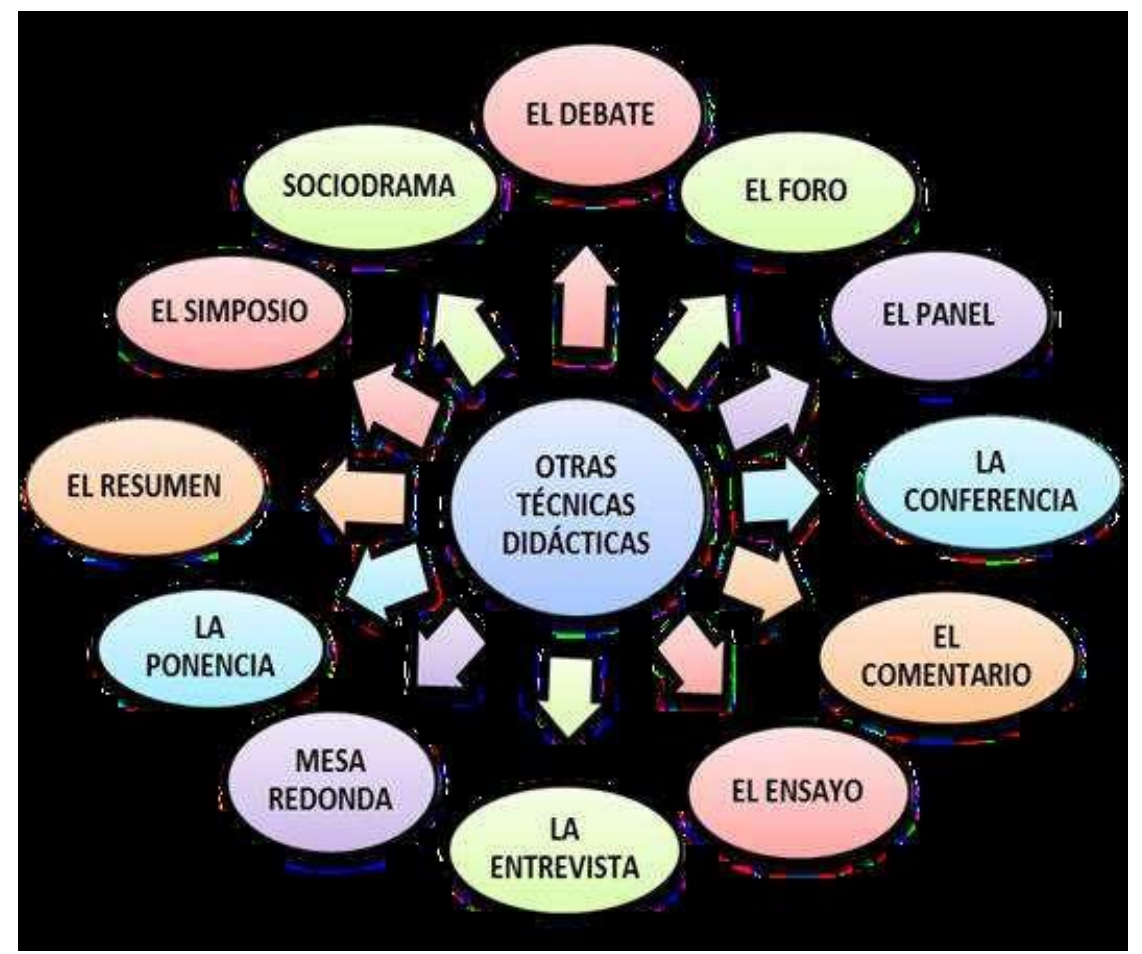

Gráfico 6. Clasificación de otras técnicas didácticas

Elaborado por: Grupo investigador

Desarrollo de habilidades con Técnicas Activas

De acuerdo a lo señalado por Esteve \& Gisbert (2011, 9(3), 55-73.), el Espacio Europeo de Educación Superior ha propiciado un nuevo marco, centrado en el estudiante y en su aprendizaje lo largo de la vida, que ofrece nuevas oportunidades formativas. Para ello, resulta necesario que la universidad replantee su modelo educativo, impulsando y estimulando la educación activa del estudiante, a través de su planificación docente e institucional. En el primer apartado de este artículo, se revisan algunos ejemplos de modelos educativos centrados en el estudiante, desde la perspectiva de la institución, el profesorado y el estudiante. Asimismo, las TIC juegan un importante papel en este cambio de paradigma. En la segunda parte de este artículo se revisa la posible contribución de estas herramientas como facilitadoras de los procesos de administración y gestión; como contenido de estudio, que permita a los estudiantes enfrentarse a los nuevos retos de la sociedad, a través 
de una adecuada alfabetización digital; y, sobre todo, como facilitadoras de nuevos modelos y estrategias de formación.

En la investigación: Desafíos y roles de la educación superior (Pedraja \& Rodríguez, 2014, págs. 156-157), enfatizan que la educación superior es tanto un bien público como un imperativo estratégico para las naciones, porque constituye la base fundamental de la investigación, la innovación y la creatividad. Por cierto, la fuente principal de la ventaja competitiva de los países, las organizaciones y las personas está en el conocimiento; el que se genera a partir de los impactos que permean desde la educación superior hacia la sociedad en su conjunto.

\section{Metodología}

La metodología que se empleó en este trabajo fue la investigación de campo, que sirvió para recopilar información en el mismo lugar objeto de estudio, el método Cuasi - experimental que permitió encontrar las posibles causas y efectos del problema, se hizo uso de técnicas de investigación como: la observación, la encuesta donde se utilizó un cuestionario estructurado con preguntas objetivas a Estudiantes y Docentes, entrevista a Autoridades de la institución que precisaron la efectividad del problema del tema de estudio, para obtener las conclusiones sobre qué aspectos fueron los más relevantes del proceso investigativo.

Esta metodología y técnicas permitieron verificar la hipótesis, sobre la escasa formación docente al no emplear un manual de técnicas activas que incide negativamente en la deficiente educación de la carrera de Comercio Exterior de la Universidad Estatal del Sur de Manabí.

\section{Resultados}

Para el desarrollo del proceso investigativo fue necesario la intervención de una población de 35 docentes y 60 estudiante, referente a la escasa aplicación de técnicas activas por los profesores en la educación de los estudiantes de la Carrera de Comercio Exterior de la Universidad Estatal del Sur de Manabí, según Ausubel el proceso de Interaprendizaje se desarrolla cuando lo que se trata de aprender se logra relacionar de forma sustantiva y no arbitraria con lo que ya conoce quien aprende, es decir, con aspectos relevantes y preexistentes de su estructura cognitiva. Esta relación o anclaje de lo que se aprende con lo que constituye la estructura cognitiva del que aprende, para Ausubel, tiene consecuencias trascendentes en la forma de abordar la enseñanza.

Los resultados de mayor relevancia de la encuesta a estudiante se detallan a continuación:

1. ¿Conoce usted a que se debe el déficit educativo que existe en la Carrera de Comercio Exterior de la Universidad Estatal del sur de Manabí? 
Tabla 1. Déficit educativo

\begin{tabular}{|l|c|c|}
\hline \multicolumn{1}{|c|}{ ALTERNATIVAS } & F & $\%$ \\
\hline $\begin{array}{l}\text { Docentes sin } \\
\text { conocimientos de técnicas } \\
\text { de enseñanza }\end{array}$ & 41 & 68 \\
\hline $\begin{array}{l}\text { Programas analíticos } \\
\text { descontextualizados }\end{array}$ & 5 & 8 \\
\hline $\begin{array}{l}\text { Malla curricular } \\
\text { desactualizada }\end{array}$ & 5 & 8 \\
\hline $\begin{array}{l}\text { Poca Promoción de la } \\
\text { Carrera }\end{array}$ & 9 & 15 \\
\hline \multicolumn{1}{|c|}{ TOTAL } & $\mathbf{6 0}$ & $\mathbf{1 0 0 \%}$ \\
\hline
\end{tabular}

Fuente: Encuesta a estudiantes carrera Comercio Exterior Elaboración: Equipo investigador

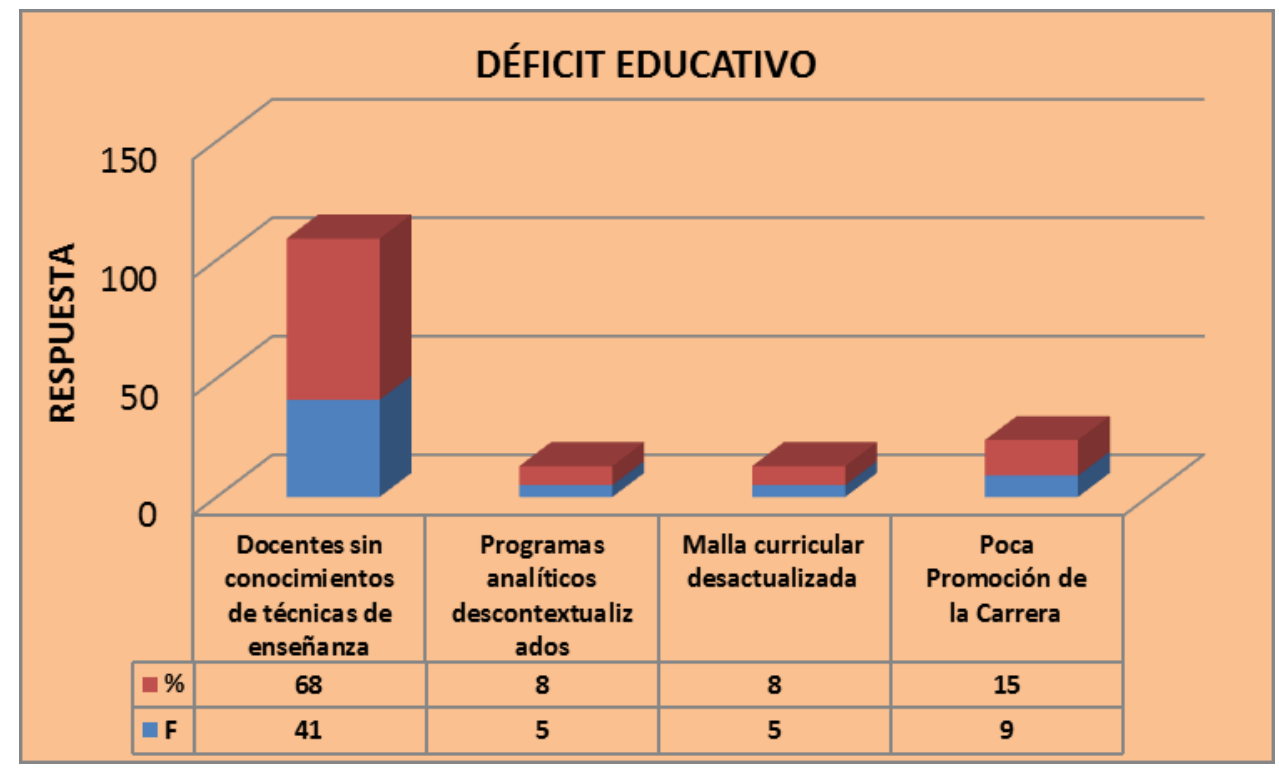

Gráfico 7. Déficit educativo

Elaboración: Equipo investigador

En correlación a la pregunta, 35 estudiantes indicaron en un $65 \%$ que el déficit educativo se debe a que los Docentes no tienen conocimientos de técnicas de enseñanza, un $17 \%$ que corresponde a 9 estudiantes indicaron que es la Poca Promoción de la Carrera que se realiza en cada semestre. Un 9\% que corresponde a un grupo de 5 estudiantes que esto se debe a Programas analíticos 
descontextualizados y a la Malla curricular desactualizada.

El desafío para las instituciones de Educación Superior es el de enfrentar un mundo en el cual los sistemas productivos están en permanente transformación y que a la vez abren nuevas perspectivas para la docencia y la investigación (Escudero Escorza, Tomás. "Enfoques Modélicos y Estrategias en la Evaluación de Centros Educativos". 1997). En esta época que vivimos hoy en día, sabemos que la educación es gratuita y que las universidades están abiertas al pueblo, pero todavía seguimos teniendo problemas, debido a que las autoridades universitarias no distribuyen adecuadamente el presupuesto de la universidad y no toman en cuenta la voz del estudiantado.

2. ¿Deduce usted que sus Docentes, conocen y aplican diversos métodos, técnicas y estrategias en su pertinencia en el marco conceptual de desarrollo de la educación universitaria actual?

Tabla 2. Aplicación de métodos, técnicas y estrategias en el trabajo docente

\begin{tabular}{|c|c|c|}
\hline ALTERNATIVAS & $\mathbf{F}$ & $\%$ \\
\hline Siempre & 8 & 14 \\
\hline A veces & 2 & 3 \\
\hline Pocas veces & 50 & 83 \\
\hline Nunca & 0 & 0 \\
\hline Total & $\mathbf{6 0}$ & $\mathbf{1 0 0}$ \\
\hline
\end{tabular}

Fuente: Encuesta a estudiantes carrera Comercio Exterior Elaboración por: Equipo investigador

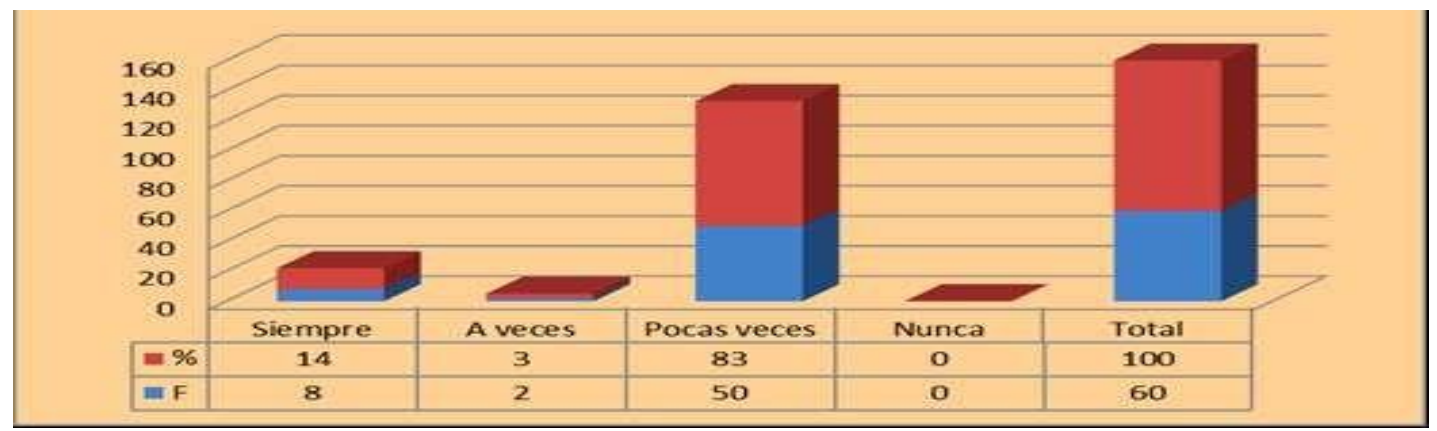

Gráfico 8. Aplicación de métodos, técnicas y estrategias en el trabajo docente

Elaboración: Equipo investigador 
En relación a la pregunta, los educandos puntualizaron en un $83 \%$ que corresponde a un grupo de 50 estudiantes que sus docentes pocas veces aplican métodos, técnicas y estrategias en el desarrollo de sus actividades de clases, un $14 \%$ que corresponde a 8 señalaron que siempre emplean métodos, técnicas y estrategias y un $3 \%$ que corresponde a 2 estudiantes detallaron que a veces usan algún métodos, técnicas y estrategias.

Un método de aprendizaje puede considerarse como un plan estructurado que facilita y orienta el proceso de aprendizaje. Se puede decir que es un conjunto de disponibilidades personales e instrumentales para la práctica formativa, deben organizarse para promover el aprendizaje (Pestalozzi, Enseñar a Pensar 1.819).

Es así que la aplicación de métodos, técnica y estrategias produzca en el profesor seguridad en sus acciones para hacer su trabajo más profesional.

3. ¿Cómo calificaría usted el uso de estrategias y técnicas activas aplicadas por los docentes de la carrera de Comercio Exterior de la Universidad Estatal del sur Manabí?

Tabla 3. Calificación a docentes del uso de estrategias y técnicas activas

\begin{tabular}{|l|c|c|}
\hline ALTERNATIVAS & F & $\%$ \\
\hline Sobresaliente & 0 & 0 \\
\hline Muy bueno & 4 & 7 \\
\hline Bueno & 5 & 9 \\
\hline Regular & 51 & 84 \\
\hline Insuficiente & 0 & 0 \\
\hline Total & $\mathbf{6 0}$ & $\mathbf{1 0 0}$ \\
\hline
\end{tabular}

Fuente: Encuesta a estudiantes carrera Comercio Exterior Elaboración: Equipo investigador 


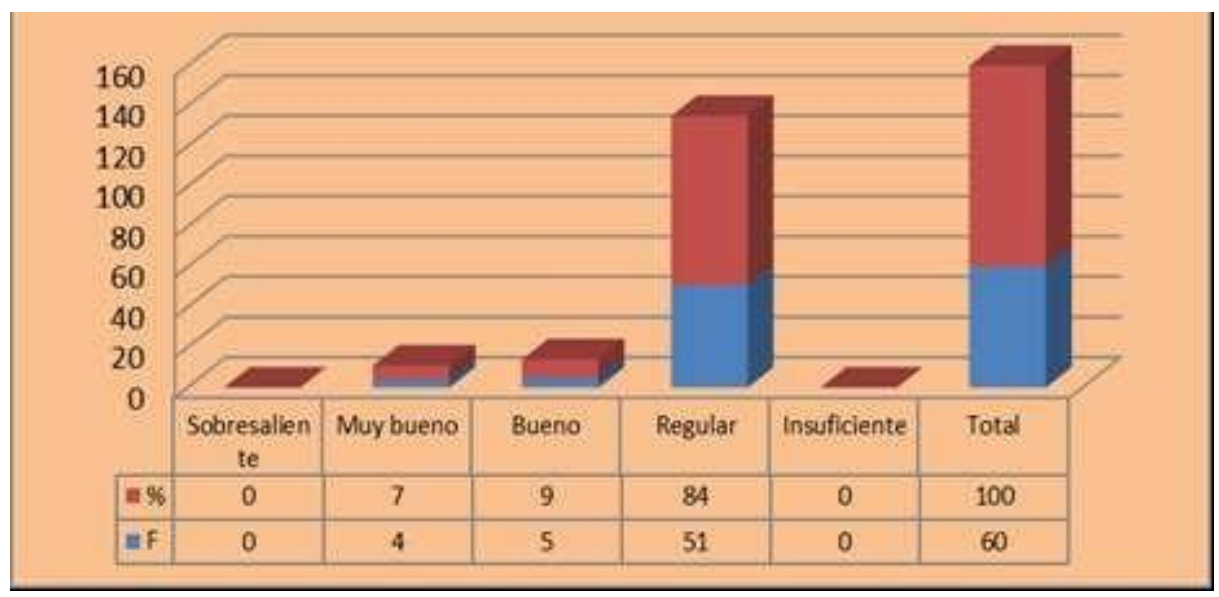

Gráfico 9. Calificación a docentes del uso de estrategias y técnicas y activas Elaboración: Equipo investigador

En la pregunta de la encuesta, los alumnos puntualizaron en un $84 \%$ que corresponde a un grupo de 51 estudiantes manifestaron que los docentes aplican de forma regular estrategias y técnicas activas en sus clases. Un $9 \%$ que corresponde a un grupo de cinco 5 señalaron que los profesores emplean estrategias y técnicas consideradas como buena y un $7 \%$ que corresponde a 4 lo consideran como muy bueno la aplicación de estrategias y técnicas.

...es necesario estimular la creatividad y la capacidad de orientación autónoma, las habilidades personales del pensamiento y también la capacidad de colaborar con los demás (Gagné, 1970).

En cambio, en los resultados de los docentes se puntualiza los siguientes:

4. ¿Considera usted que la débil formación profesional de los actores educativos se debe a que el modelo educativo está atrasado?

Tabla 4. Débil formación por el modelo educativo atrasado

\begin{tabular}{|l|c|c|}
\hline ALTERNATIVAS & $\begin{array}{c}\text { FRECUENCIA } \\
\text { ABSOLUTA }\end{array}$ & $\begin{array}{c}\text { FRECUENCIA } \\
\text { RELATIVA }\end{array}$ \\
\hline Sí & 20 & $57 \%$ \\
\hline No & 15 & $43 \%$ \\
\hline \multicolumn{1}{|c|}{ TOTAL } & 35 & $100 \%$ \\
\hline
\end{tabular}

Fuente: Encuesta a Docentes carrera Comercio Exterior

Elaboración: Equipo investigador 


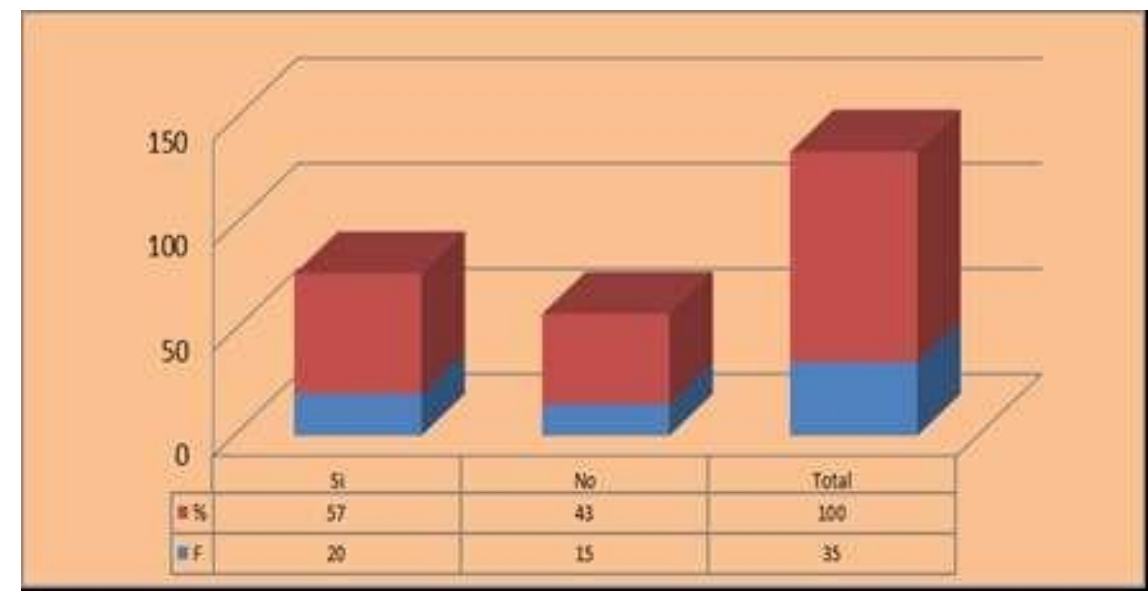

Gráfico 10. Débil formación por el modelo educativo atrasado

Elaboración: Equipo investigador

En relación a la pregunta se observa que los docentes en un $57 \%$ consideran que Sí, lo que corresponde a un grupo de 20 docentes y 15 con $43 \%$ que No. Se puede deducir que existe una desvalorización de la profesión docente y de la educación como campo de investigación y acción, es decir las tradicionales instituciones de educación, ya sean presenciales o a distancia, tienen que reajustar sus sistemas de distribución y comunicación.

De esta manera surge la compulsión al cambio, al mejoramiento de las debilidades como un rasgo que caracteriza el discurso de la innovación. Lejos se está de pensar que toda propuesta de cambio realmente le imprime un rumbo diferente al trabajo cotidiano que se realiza en las aulas.

5. ¿Cómo calificaría usted el uso de Estrategias y Técnicas activas aplicadas para un mejor aprendizaje significativo?

Tabla 5. Aprendizaje significativo por el uso de Estrategias y técnicas activas

\begin{tabular}{|l|c|c|}
\hline ALTERNATIVAS & $\begin{array}{c}\text { FRECUENCIA } \\
\text { ABSOLUTA }\end{array}$ & $\begin{array}{c}\text { FRECUENCIA } \\
\text { RELATIVA }\end{array}$ \\
\hline Excelente & 0 & $0 \%$ \\
\hline Muy buenas & 30 & $86 \%$ \\
\hline Buenas & 5 & $14 \%$ \\
\hline Regulares & 0 & $0 \%$ \\
\hline Malas & 0 & $0 \%$ \\
\hline \multicolumn{1}{|c|}{ TOTAL } & $\mathbf{3 5}$ & $\mathbf{1 0 0 \%}$ \\
\hline
\end{tabular}

Fuente: Encuesta a Docentes carrera Comercio Exterior Elaboración: Equipo investigador 


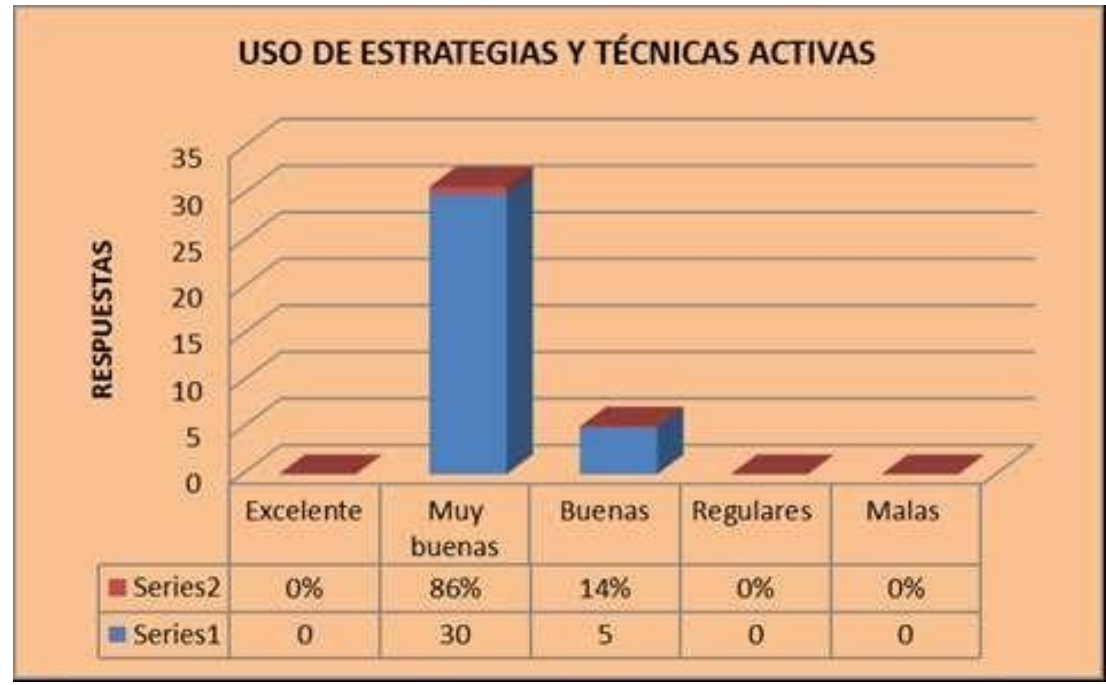

\section{Gráfico 11. Aprendizaje significativo por el uso de Estrategias y técnicas activas}

Elaboración: Equipo investigador

En concordancia a la presente pregunta los docentes expresaron en un $86 \%$ que corresponde a un grupo de 20 encuestados, indicaron que es muy bueno el uso de Estrategias y Técnicas activas aplicadas para un mejor aprendizaje significativo y un $14 \%$ manifestaron que es bueno.

León Tolstoi (Rusia, 1828-1910), uno de los más grandes novelistas del siglo XIX, en 1859 abrió en su finca de Iásnaia Poliana una escuela para los hijos de sus campesinos basada en el principio que dice así: mientras menor sea la constricción requerida para que los jóvenes aprendan, mejor será el uso de estrategias y técnicas.

Las estrategias y técnicas permiten ordenar el proceso educativo, dándole una secuencia a las actividades didácticas para el desarrollo del proceso enseñanza aprendizaje. Cuando se emplea un método y conscientemente se sabe cuál es ese método; los requisitos, los condicionamientos, las estrategias, los momentos, su empleo hace más profesional el aprendizaje significativo en las instituciones educativas superiores o Universidades.

6. ¿Considera usted que se debe de aplicar técnicas activas para lograr una verdadera educación activa? 
Tabla 6. Aplicación de Técnicas activas

\begin{tabular}{|c|c|c|}
\hline ALTERNATIVAS & $\begin{array}{c}\text { FRECUENCIA } \\
\text { ABSOLUTA }\end{array}$ & $\begin{array}{c}\text { FRECUENCIA } \\
\text { RELATIVA }\end{array}$ \\
\hline Sí & 35 & $100 \%$ \\
\hline No & 0 & $0 \%$ \\
\hline TOTAL & 35 & $100 \%$ \\
\hline
\end{tabular}

Fuente: Encuesta a Docentes carrera Comercio Exterior

Elaboración: Equipo investigador

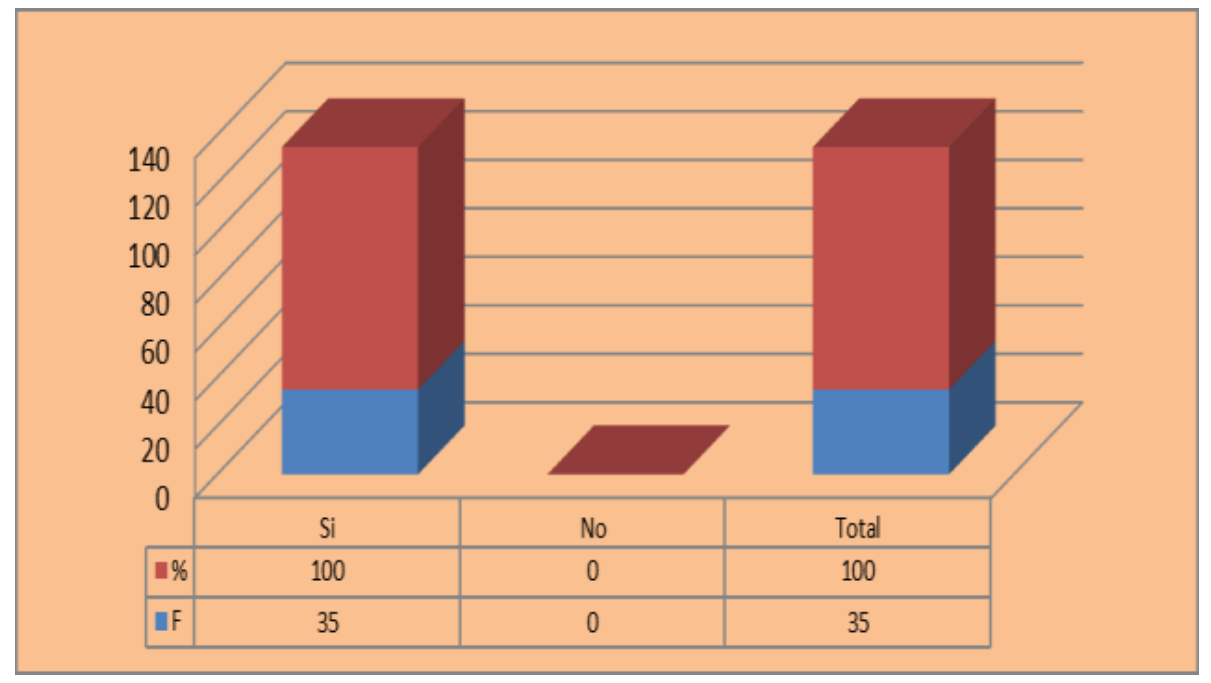

Gráfico 12. Aplicación de Técnicas activas

Elaboración: Equipo investigador

En correlación a la presente interrogante, los docentes señalaron en un $100 \%$ que corresponde a un grupo de 35 docentes que sí aplican técnicas activas para lograr una educación de calidad a sus estudiantes.

Las técnicas activas para el desarrollo del trabajo docente educativo, se debe a la excelente formación del profesor.

\section{Conclusiones}

Existe una clara evidencia que los docentes para el desarrollo de sus actividades educativa, no han estado aplicando una metodología activa que propicie en el estudiante un aprendizaje significativo, tal como lo exige la sociedad y la exigencia de la educación moderna. La enseñanza moderna es un producto de un determinado 


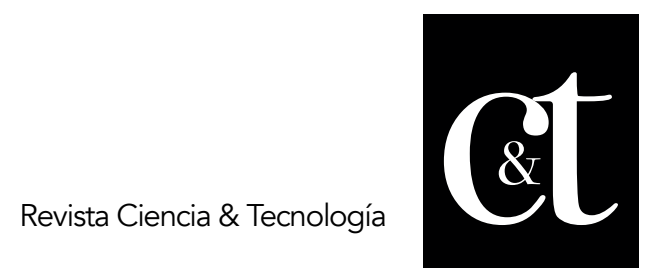

No. 17, 31 de enero de 2018

ISSN impreso: 1390 - 6321

proceso, ya que tiene como misión constituir un proyecto susceptible de preparar al profesional para la sociedad en la que está llamado a evolucionar, lo que hace meditar la importancia del proceso del sistema educativo.

Considerando los resultados y validando los objetivos propuesto en la investigación, se hizo necesario que el docente utilice técnicas activas, para lo cual se planteó como alternativa de solución un Manual de Técnicas de aprendizaje para que los docentes apliquen en cada una de sus clases, y así mejorar el rendimiento de los educandos, ya que una capacitación especial en la formación del docente, es aquella destinada a hacer frente a las necesidades específicas de los estudiantes.

\section{Referencias Bibliográficas}

Apple, M. \& Jungck, S. (1990). No hay que ser maestro para la enseñanza esta unidad: la enseñanza, la tecnología y el control en el aula. Revista de Educación, No 291: 149 - 172. Madrid.

Aznar, P. (1995). El estudio de casos como técnica de simulación: aplicaciones educativas, en López-Barajas, E. y Montoya, J.M. (eds.): El estudio de casos: fundamentos y metodología. Madrid: UNED.

Benito, A. , \& Cruz, A. (2005). Nuevas claves para la docencia universitaria en el Espacio Europeo de Educación Superior. Madrid: Narcea.

Carr, W. (1990). Hacia una ciencia crítica de la educación. Barcelona: Ed. Laertes.

Carrera, X. (2006). Aprendre a través dels jocs de simulació. Lleida: ICE - Edicions i Publicacions, Universitat de Lleida.

Comenius, J.A. (2009). La Didáctica Basada en Datos Empíricos y Científicos de la Educación.

Davini, M. C. \& Alliaud, A. (1995). Los maestros del siglo XXI, Miño y Dávila Editores, Buenos Aires.

Esteve, M. F., \& Gisbert, C. M. (2011). El nuevo paradigma de aprendizaje y nuevas tecnologías. REDU. Revista de Docencia Universitaria 9(3): 55-73.

Flores, O. R. (1994). Hacia Una Pedagogía del Conocimiento, Santa Fe de Bogotá: McGraw Hill.

Gagné, R. (1970). Las condiciones del aprendizaje. Aguilar.

Hornilla, T. (1999). Formación del profesorado universitario y calidad de la enseñanza. Servicio de Publicaciones.

Instituto Tecnológico Monterrey (2010). Técnicas didácticas activas. Centro Virtual de Técnicas Didácticas. Obtenido de http://www.sistema.itesm.mx/va/dide2/tecnicas_didacticas/abp/qes.htm

López, M. (2003). Técnicas de Enseñanza en Grupo. México: Trillas.

Madrid, J.M. (2005). La formación y la evaluación docente del profesorado universitario ante el espacio europeo de educación superior. Educativo Siglo XXI. 


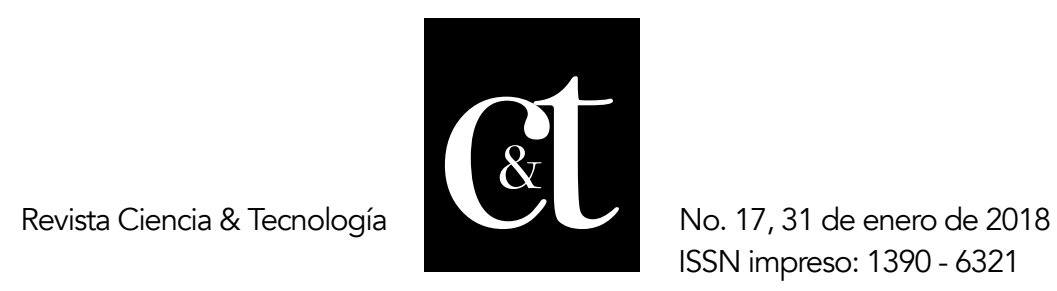

Martín, E. (2013). Jugando a hacer Historia: los juegos de simulación como recurso didáctico. Madrid: Edisa Madrid.

Pazmiño, L. (2011). Técnicas innovadoras para la enseñanza y el aprendizaje (Primera ed.). Ecuador: Zurita.

Pedraja, R. L., \& Rodríguez, P. E. (2014). Desafíos y roles de la educación superior. Ingeniare. Revista chilena de ingeniería, 22(2).

Prieto, L. (2006). Aprendizaje activo en el aula universitaria: el caso del aprendizaje basado en problemas, en Miscelánea Comillas. Revista de Ciencias Humanas y Sociales, Vol.64. Núm.124, 173-196.

Rosler, R. (2007). Técnicas de Enseñanza (Enseñar a Enseñar). Rev.Chil.Neurocirug, $28,85-88$.

Sánchez de Magurno, J. (1998). La formación docente en debate. Buenos Aires: Academia Nacional de Educación.

Tobón, S. (2005). "Técnicas Didácticas Activas" equipo formadores regionales Boyacá Sena. Instituto Tecnológico y de Estudios Superiores de Monterrey. Monterrey. 